\title{
Thermal poling induced second-order nonlinearity in femtosecond- laser-modified fused silica
}

\author{
Honglin An, ${ }^{1, a)}$ Simon Fleming, ${ }^{1}$ Benjamin W. McMillen, ${ }^{2}$ Kevin P. Chen, ${ }^{2}$ and \\ David Snoke ${ }^{3}$ \\ ${ }^{1}$ Optical Fibre Technology Centre, University of Sydney, 206 National Innovation Centre, Australian \\ Technology Park, Eveleigh, New South Wales 1430, Australia \\ ${ }^{2}$ Department of Electrical and Computer Engineering, University of Pittsburgh, Pennsylvania 15261, USA \\ ${ }^{3}$ Department of Physics, University of Pittsburgh, Pennsylvania 15261, USA
}

(Received 9 May 2008; accepted 29 July 2008; published online 15 August 2008)

\begin{abstract}
Thermal poling was utilized to induce second-order nonlinearity in regions of fused silica modified by $771 \mathrm{~nm}$ femtosecond laser pulses. With second-harmonic microscopy, it was found that the nonlinearity in the laser-modified region was much lower than that in nonmodified regions. This is attributed to a more rigid glass network after irradiation by the femtosecond laser pulses and/or lack of mobile alkali ions. Measurement of the distribution of chemical elements in the femtosecond-laser-modified region in a soda lime glass revealed a lower level of sodium ions. (C) 2008 American Institute of Physics. [DOI: 10.1063/1.2973149]
\end{abstract}

Thermal poling has been widely used for inducing large second-order optical nonlinearity (SON) in amorphous silicate glasses. ${ }^{1}$ The poling process relies on the creation of a strong electric field under the anode surface through migration of mobile charges under the influence of the applied electric field. ${ }^{2,3}$ The resultant electric field is frozen in the glass after the poled sample cools down to room temperature, generating an effective SON through a third-order nonlinear process, $\chi^{(2)}=3 \chi^{(3)} E_{\text {frozen }}$. Poled glasses and waveguides are potential materials for many nonlinear optical applications. So far, second-harmonic ( $\mathrm{SH})$ generation has already been realized in poled optical fibers and planar waveguides. ${ }^{4,5}$ Electro-optic (EO) switches and modulators based on thermally poled planar waveguides and fibers have also been demonstrated and tested in real transmission systems. ${ }^{6-8}$

In the past several years, femtosecond laser pulses have been successfully used to directly write microstructures in transparent materials through a multiphoton absorption process, and low-loss waveguides have been obtained in optical glasses. ${ }^{9,10}$ It would be scientifically interesting and technologically important to investigate the feasibility of inducing SON in these femtosecond-laser-written optical waveguides. Thermal poling of a silica glass premodified by femtosecond laser irradiation was first reported in 2002, with an increase in SON being found in the structurally modified glass region. ${ }^{11}$ Recently, an EO waveguide modulator fabricated in fused silica by a combination of femtosecond laser direct writing and thermal poling has been demonstrated with an estimated effective EO coefficient of $\sim 0.17 \mathrm{pm} / \mathrm{V}$ for the TE mode. ${ }^{12}$ Apart from these two, relevant reports are still scarce. In this letter, we report our result of characterizing the SON in thermally poled fused silica with microstructures written by femtosecond laser pulses. Direct visualization of the SON distribution reveals a substantially different behavior from the previously reported results.

The glass samples used in our experiment are Corning 7980 synthetic flame-fused silica plates. Parallel lines of mi-

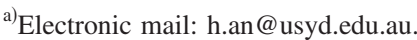

crostructures were written in the bulk of the fused silica plates by scanning with a focused beam from a mode-locked Ti:sapphire laser operating at $771 \mathrm{~nm}$ with a repetition rate of $250-500 \mathrm{kHz}$ and pulse duration of $150 \mathrm{fs}$. The pulse energy ranged from 160 to $300 \mathrm{~nJ} /$ pulse and the scanning speed varied from 50 to $500 \mu \mathrm{m} / \mathrm{s}$. All written lines are in a plane parallel to the surface of the silica plate, $\sim 12-25 \mu \mathrm{m}$ beneath the surface. For silica plates with modified structures more than $15 \mu \mathrm{m}$ beneath the surface, hydrofluoric acid etching was used to make the plates thinner until the written structures were $\leq 15 \mu \mathrm{m}$ beneath the surface to ensure an effective overlap between the laser-written structures and the thermal poling induced nonlinear region. Typical white-light optical microscopy images of the laser-written structures are shown in Fig. 1. The upper and lower filamentary tracks in Fig. 1 are written with $240 \mathrm{~nJ}$ pulses at $250 \mathrm{kHz}$ with scanning speeds of 100 and $75 \mu \mathrm{m} / \mathrm{s}$, respectively. The end-face image clearly indicates that waveguiding structures have been successfully fabricated in the silica plate. The dark region behind the light-guiding region is caused by significant structural irregularities in the laser-modified region, probably caused by microexplosions. ${ }^{13}$ Thermal poling was carried out in air with pressed-on electrodes at $3.5 \mathrm{kV}$ and $280{ }^{\circ} \mathrm{C}$ for 70-100 min - the exact poling duration being determined by the depth of the written microstructures in the silica plates. After poling, the spatial profile of the SON was investigated by directly visualizing the distribution of the generated $\mathrm{SH}$ signals from the poled samples using $\mathrm{SH}$ microscopy. ${ }^{14}$ In
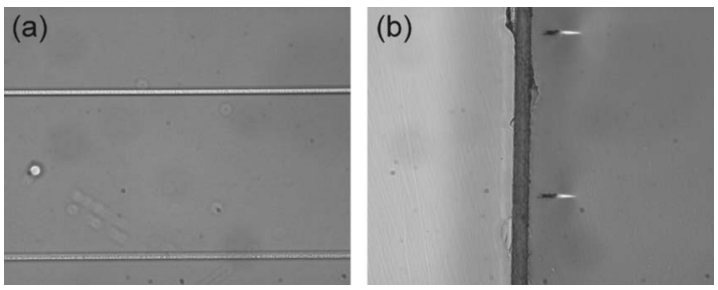

FIG. 1. Light microscopy images of the laser-written filamentary structures in Corning 7980 silica plates. (a) Top view and (b) side view. The line spacing is $100 \mu \mathrm{m}$. The femtosecond laser pulses propagate from left to right in (b). 

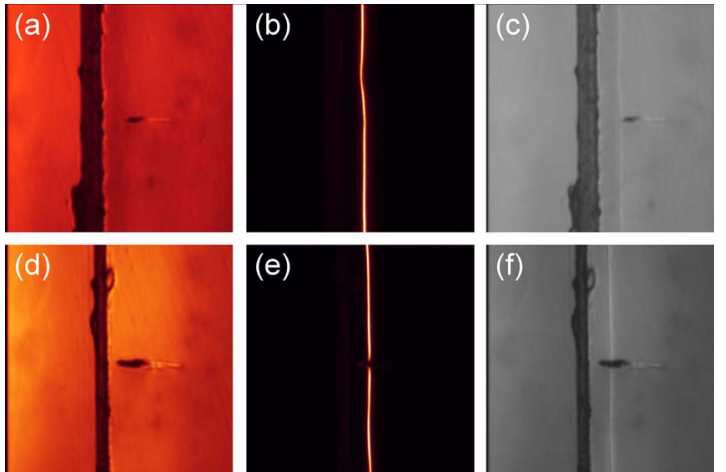

FIG. 2. (Color online) Distribution of SH light in femtosecond-lasermodified Corning 7980 silica thermally poled for $70 \mathrm{~min}$. (a) and (d) are ordinary transmission images, (b) and (e) are SH images, and (c) and (f) are overlay images of the corresponding ordinary transmission and SH images.

microscopy specimen preparation, a microscope cover slip was usually affixed to the anode side of the poled silica plates for support and protection during sample grinding and polishing. Then the whole block was cleaved through the poled region to expose two cross sections that are parallel to the applied poling field. Finally, the two cross sections were polished to an optical finish for the SH microscopy observation. In the measurement, the samples were rotated so that the polarization direction of the fundamental laser beam was parallel to the poling direction to utilize the larger nonlinearity component $d_{33}$. The transverse spatial resolution of the SH microscopy is estimated to be $\sim 0.4 \mu \mathrm{m}$.

Typical SH micrographs of the silica plates poled for 70 min are shown in Fig. 2. The modification line in Figs. 2(a)-2(c) is written with $160 \mathrm{~nJ}$ pulses at $250 \mathrm{kHz}$ and $150 \mu \mathrm{m} / \mathrm{s}$, while that in Figs. 2(d)-2(f) is written with 160 $\mathrm{nJ}$ pulses at $500 \mathrm{kHz}$ and $100 \mu \mathrm{m} / \mathrm{s}$. In all the poled plates, the induced SON is found to be localized mainly in a narrow layer, $\sim 9.4 \mu \mathrm{m}$ beneath the anode surface. In Fig. 2(a), the SON layer has not reached the laser-modified region and is continuous and uniform in signal strength, as observed in our earlier work. ${ }^{13}$ In Fig. 2(d), the laser-modified region is closer to the surface, and the SON layer now intersects the dark part of the modified region. The original continuous SON layer is now broken into two sections, with no $\mathrm{SH}$ signal coming from the modified region. Presumably this is due to the microexplosion induced structural irregularities, ${ }^{14}$ which make it impossible to create SON in this region. For all samples poled for $70 \mathrm{~min}$, the SON layer has not reached the waveguiding region. The SON magnitude, $d_{33} \sim 0.07$ $-0.1 \mathrm{pm} / \mathrm{V}$, is also lower than that in fused quartz silica.

To better overlap the nonlinear region with the laserwritten waveguides, a longer poling duration of $100 \mathrm{~min}$ was chosen. Before poling, some silica plates were cut into two half-sections, one of which had an additional processing step. As alkali ions are known to play an important role in poling, it was suspected that their scarcity in the synthetic Corning 7980 silica might be responsible for the smaller SON achieved. Thus one-half of each plate was first annealed in air at $600{ }^{\circ} \mathrm{C}$ for $\sim 4 \mathrm{~h}$ in an attempt to introduce and redistribute some alkali ions to the annealed plates. Both halves were then identically poled. Typical SH micrographs from the polished cross sections of these samples are shown in Fig. 3. In all the images, the annealed and unannealed halves of the same sample are affixed back to back-the
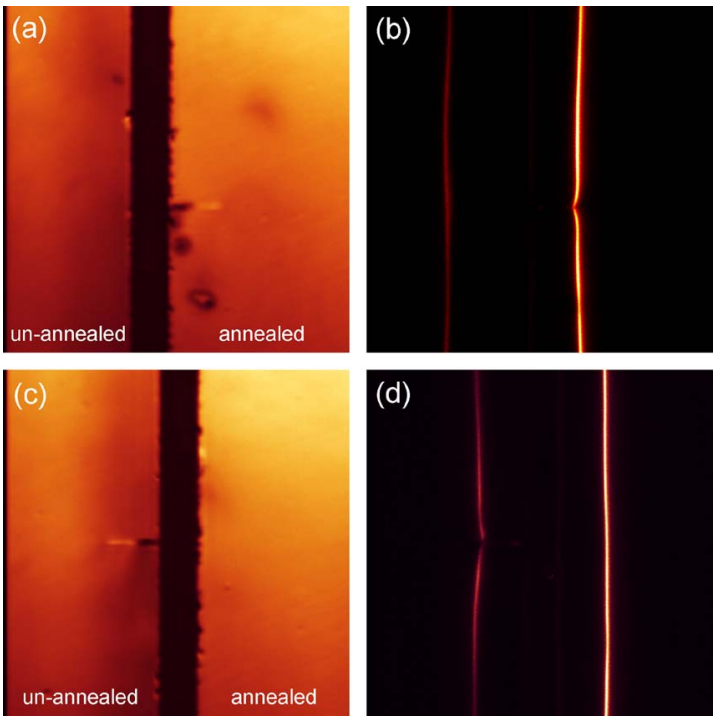

FIG. 3. (Color online) Distribution of SH light in femtosecond-lasermodified Corning 7980 silica thermally poled for $100 \mathrm{~min}$. (a) and (c) are ordinary transmission images, while (b) and (d) are SH images.

left-side section being unannealed and the right side annealed. By measuring the positions of the waveguides and SON layers, it is clear that the SON layer has now reached the waveguides in both samples, being $\sim 3.1 \mu \mathrm{m}$ into the unannealed waveguide and $\sim 2.7 \mu \mathrm{m}$ into the annealed waveguide. The SON layer is weaker in the waveguiding regions than that outside the laser-modified region in both samples. The $d_{33}$ in the waveguide was measured to be at least 1.9 times lower than that outside the waveguide. Some other interesting effects can also be observed. The SON layer around the waveguides seems to lag behind that farther away from the laser-modified region, indicating a decreased ion mobility in this region, perhaps a result of the glass network becoming denser due to laser irradiation. The SON layers well away from the laser-modified region are all straight and uniform in intensity. Another interesting feature is that the SH signal layer in the annealed samples is much brighter than that in the unannealed samples. The ratio of $d_{33}$ in the two samples was estimated to be in the range of 1.6-2.5. This increase is attributed to a higher concentration of mobile alkali ions in the annealed sample, with surface contamination due to handling as the primary source of alkali ions.

Our findings are in contrast to the previously published result, ${ }^{11}$ where an enhancement in SON was found in the laser-modified region. However, a direct comparison cannot be made as the silica types and femtosecond laser irradiation conditions are different.

We tentatively attribute the weaker SON in the lasermodified region to the more compact glass network there. The femtosecond-laser-written waveguide is a direct result of the denser glass structure. Lower mobility for alkali ions and other charges within this densified glass region is expected, making it more difficult for relevant ions to either enter or leave this modified region to form the frozen-in space-charge field. ${ }^{2,3}$ The net result is a weak space-charge field and a corresponding weak SON. The resulting densified silica network might be accompanied by a further reduction in alkali ion density in the laser-modified region. To test this hypothesis, a soda lime glass was chosen and the distribution of alkali ions in the modified region written by a Spitfire Ti:sap- 


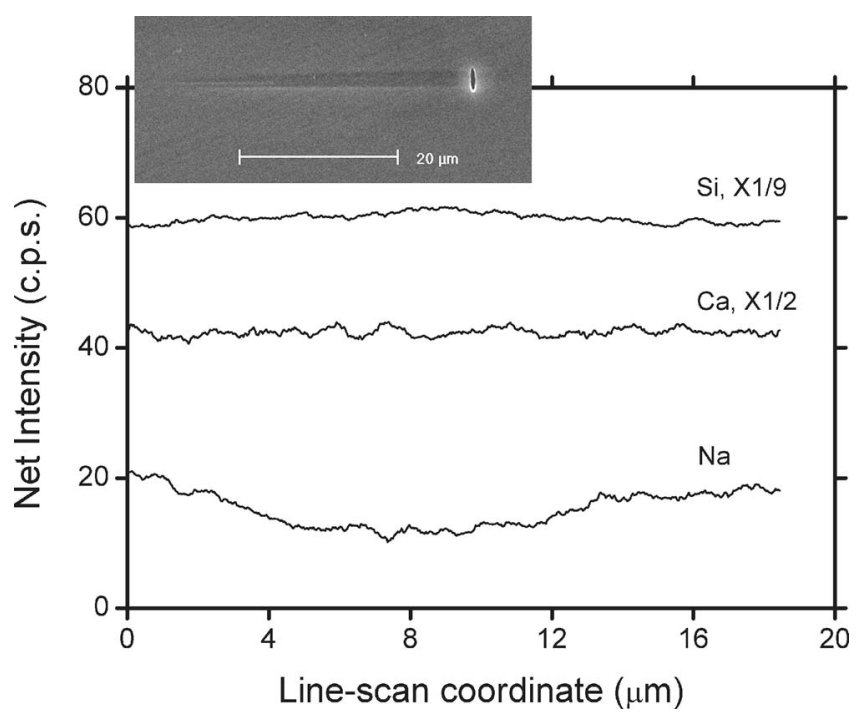

FIG. 4. Distribution of chemical elements within the modified region of soda lime glass by femtosecond laser irradiation. Inset is a SEM image of the modified region.

phire laser $(800 \mathrm{~nm}$ wavelength, $1 \mathrm{kHz}$ repetition rate, $120 \mathrm{fs}$ pulse duration, $\sim 1.2 \mu \mathrm{J}$ pulse energy, $50 \mu \mathrm{m} / \mathrm{s}$ scanning speed, and 0.25 numerical aperture focusing lens) was measured with an energy dispersive x-ray spectrometry (EDS). No attempt was made on fused silica samples because the level of alkali ions is beyond the limit of the EDS technique. The result from a line scan across the modified region is shown in Fig. 4. For easy identification of the modified region under a scanning electron microscope (SEM), the polished surface was slightly etched in diluted hydrofluoric acid. $\mathrm{Si}$ is shown as a reference. It is clear that the concentration of $\mathrm{Na}$ decreased in the laser-modified region while $\mathrm{Ca}$ stays almost unchanged. Similar results have been reported in a $\mathrm{PbS}$-doped silicate glass irradiated with femtosecond laser pulses. ${ }^{15}$ These results provide indirect support to the hypothesis of a lower level of $\mathrm{Na}$ in the laser-modified region in fused silica. Further experiments are needed to fully resolve this issue.

In conclusion, we have studied the distribution of the induced SON in femtosecond-laser-modified regions in
Corning 7980 fused silica. It has been found that either there is no SON or there is a very weak SON in the laser-modified region. The effect is attributed to the lower mobility of alkali ions, probably due to the denser glass network in the modified region. These results have important implications for constructing EO modulators and switches in waveguides fabricated by femtosecond laser direct writing technique.

The authors acknowledge the facilities as well as scientific and technical assistance from the staff in the Australian Microscopy and Microanalysis Research Facility (AMMRF) at the Electron Microscope Unit, University of Sydney. The authors also thank Professor Yan Li and Professor Qihuang Gong of the School of Physics, Peking University, People's Republic of China, for providing the laser-modified soda lime glass samples used in this experiment. This research was supported in part under the Australian Research Council's Discovery funding scheme (Project No. DP0774404) and by the U. S. National Science Foundation (Grant Nos. CMMI 0556086 and DMI 0216785).

${ }^{1}$ R. A. Myers, N. Mukherjee, and S. R. J. Brueck, Opt. Lett. 16, 1732 (1991).

${ }^{2}$ P. G. Kazansky and P. St. J. Russell, Opt. Commun. 110, 611 (1994)

${ }^{3}$ T. G. Alley, S. R. J. Brueck, and R. A. Myers, J. Non-Cryst. Solids 242, 165 (1998).

${ }^{4}$ H. Y. Chen, J. S. Sue, Y. H. Lin, and S. Chao, Opt. Lett. 28, 917 (2003).

${ }^{5}$ A. Canagasabey, C. Corbari, Z. Zhang, P. G. Kazansky, and M. Ibsen, Opt. Lett. 32, 1863 (2007)

${ }^{6}$ M. Abe, T. Kitagawa, K. Hattori, A. Himeno, and Y. Ohmori, Electron. Lett. 32, 893 (1996).

${ }^{7} \mathrm{~J}$. Li, N. Myrén, W. Margulis, B. Ortega, G. Puerto, D. Pastor, J. Capmany, M. Belmonte, and V. Pruneri, IEEE Photon. Technol. Lett. 17, 2571 (2005)

${ }^{8}$ O. Tarasenko and W. Margulis, Opt. Lett. 32, 1356 (2007).

${ }^{9}$ K. M. Davis, K. Miura, N. Sugimoto, and K. Hirao, Opt. Lett. 21, 1729 (1996).

${ }^{10}$ C. B. Schaffer, A. Brodeur, J. F. García, and E. Mazur, Opt. Lett. 26, 93 (2001)

${ }^{11}$ C. Corbari, J. D. Mills, O. Deparis, B. G. Klappauf, and P. G. Kazansky, Appl. Phys. Lett. 81, 1585 (2002).

${ }^{12}$ G. Li, K. A. Winick, A. A. Said, M. Dugan, and P. Bado, Opt. Lett. 31, 739 (2006).

${ }^{13}$ E. N. Glezer and E. Mazur, Appl. Phys. Lett. 71, 882 (1997).

${ }^{14}$ H. An, S. Fleming, and G. Cox, Appl. Phys. Lett. 85, 5819 (2004).

${ }^{15}$ N. Takeshima, Y. Kuroiwaa, Y. Naritaa, S. Tanakaa, and K. Hiraob, Opt. Express 12, 4019 (2004). 
Applied Physics Letters is copyrighted by the American Institute of Physics (AIP). Redistribution of journal material is subject to the AIP online journal license and/or AIP copyright. For more information, see http://ojps.aip.org/aplo/aplcr.jsp 